\title{
The Influence of Axial Compression Ratio on the Seismic Behavior of RC Frame Column
}

\section{Liu Yang, Gong Maosheng*, Zuo Zhanxuan}

Key Laboratory of Earthquake Engineering and Engineering Vibration, Institute of Engineering Mechanics, China Earthquake Administration, Harbin, China

\author{
Email address: \\ 1507879615@qq.com (Liu Yang), gmshiem@163.com (Gong Maosheng), zuozhanxuan@sina.com (Zuo Zhanxuan) \\ ${ }^{*}$ Corresponding author
}

\section{To cite this article:}

Liu Yang, Gong Maosheng, Zuo Zhanxuan. The Influence of Axial Compression Ratio on the Seismic Behavior of RC Frame Column. Science Discovery. Vol. 9, No. 4, 2021, pp. 178-183. doi: 10.11648/j.sd.20210904.18

Received: April 27, 2021; Accepted: June 6, 2021; Published: June 9, 2021

\begin{abstract}
In order to analyze the quantitative influence of the axial compression ratio on the seismic performance of reinforced concrete $(\mathrm{RC})$ frame column, this paper carried out the numerical analysis of the quasi-static simulation of a RC frame column and analyzed its hysteresis performance, bearing capacity, stiffness degradation, energy consumption capacity and ductility capacity under different axial compression ratios, based on OpenSees finite element software, considering the buckling and fatigue damage model. The results show that the smaller the axial compression ratio, the fuller the hysteresis curve and the slower the stiffness degradation of the column. The ultimate bearing capacity of the column increases by $24.6 \%$ when the axial compression ratio increases from 0.3 to 0.8 , but bearing capacity decreasing and stiffness degradation is faster and faster. When the deformation does not exceed the ultimate displacement, the equivalent viscous damping at each displacement level of high axial compression ratio column is greater than that of the low axial compression ratio, but the total hysteretic energy decreases about $64.2 \%$ at maximum amplitude. The ultimate displacement gradually decreases with the increase in the axial compression ratio, and the ductility factor decreases about $55.9 \%$ at maximum amplitude. The results can be referenced by the seismic design and analysis of RC frame.
\end{abstract}

Keywords: Reinforced Concrete Column, Axial Compression Ratio, Seismic Behavior, Quasi-static Simulation, Constitutive Model

\section{轴压比对 RC框架柱抗震性能影响研究}

刘洋, 公茂盛", 左占宣

地震工程与工程振动重点实验室, 中国地震局工程力学研究所, 哈尔滨, 中国

邮箱

1507879615@qq.com（刘洋），gmshiem@163.com（公茂盛）, zuozhanxuan@sina.com（左占宣）

摘要: 为了分析轴压比对钢筋混凝土（RC）框架柱抗震性能的定量影响, 本文基于OpenSees有限元软件建模, 采用考 虑屈曲和疲劳损伤的模型, 对某RC框架结构柱进行了拟静力数值模拟, 分析了不同轴压比下柱的滞回性能、承载能力、 刚度退化、耗能能力以及延性能力。分析结果表明: 轴压比越小, 柱的滞回曲线更饱满, 刚度退化越慢; 当轴压比从 0.3 增大到 0.8 时, 柱极限承载力增大 $24.6 \%$, 但承载力下降和刚度退化的越来越快; 在未超过极限变形时, 高轴压比下 柱单圈等效粘滞阻尼比更大, 但总累积耗能最大降低 $64.2 \%$; 随着轴压比不断增大, 柱极限位移逐渐降低, 延性系数 最大降低 $55.9 \%$ 。结果可供 $\mathrm{RC}$ 框架结构抗震设计及分析等参考。 
关键词: 框架柱, 轴压比, 抗震性能, 拟静力模拟, 本构模型

\section{1. 引言}

国内外历次大地震震害经验表明，建筑结构倒塌是造 成经济损失和人员伤亡的最主要原因。钢筋混凝土 (RC) 框架结构在世界范围内应用广泛, 在多次地震中也发生了 严重破坏甚至倒塌, RC框架结构发生严重破坏的原因之 一是作为主要承重构件的框架柱变形和耗能能力不足, 导 致框架柱在地震荷载下发生破坏。

针对RC框架结构抗震性能及抗倒塌能力, 国内外学 者开展了广泛的研究。陆新征等 [1]为研究汶川地震中 $R C$ 框架结构地震破坏机理, 开展了 $\mathrm{RC}$ 框架整体结构、节点 及轴压比不同的边柱和中柱的拟静力倒塌试验, 并将试验 结果进行了公开, 为检验结构及构件数值模拟分析方法提 供了基础。Yao等[2]研究轴压比的增大对超高强钢筋混凝 土框架结构抗震性能的影响。观察到随着轴压比从 0.5 增 大到 0.75 和 0.9 , 框架柱破坏模式从弯曲破坏变为弯曲粘结 破坏, 并进一步扩展为弯曲䢃裂破坏。轴压比不断增大, 伴随着强度、刚度和耗能能力的变化, 框架结构的抗震性 能降低。

白巨巨等[3]研究不同轴压比RC柱一钢梁组合件抗震 性能, 得出在一定范围内, 提高轴压比可以增大组合件承 载力, 在高轴压比下, 承载力略微降低。轴压比不断增大, 组合件等效黏滞系数总体呈增大趋势。蔡健等[4]分析轴压 比对组合剪力墙抗震性能的影响, 完成了 5 个缩尺模型试 件的拟静力试验。发现轴压比为 $0.3 \sim 0.9$ 的范围内, 试件 承载力随轴压比的增大而提高, 但高轴压比时其变形和滞 回能力有所降低。

冯宝锐[5]对 9 根轴压比分别为 $0.69 、 0.89 、 0.97 、 1.05$ 等的RC框架柱进行了抗震性能试验研究, 建立了框架柱 在地震作用下抗震性能点及损伤状态特征点与弹塑性转 角的关系, 为判别柱的损伤状态提供了参考依据。得出抗 震等级相同的RC框架柱试件, 轴压比高的框架柱的破坏 程度比轴压比低的框架柱的破坏程度严重的结论。

Sun等[6]对玻璃纤维聚合物增强RC柱进行拟静力试 验, 将轴压比从 0.3 增大到 0.45 , 再增大到 0.6 。柱的破坏 模式从弯剪破坏变为弯曲破坏, 轴压比大于 0.45 为弯曲破 坏。柱极限承载力分别增加 $8.42 \%$ 和 $21.49 \%$, 极限变形分 别减少 $5.56 \%$ 和 $11.76 \%$, 累积耗能分别降低 $43.56 \%$ 和 $7.79 \%$ 。

Liu等[7]使用有限元软件ABAQUS对纤维增强RC柱 进行数值建模, 将轴压比为 $0.15 、 0.25$ 的柱模拟结果与试 验现象进行对比验证, 将为轴压比 $0.2 、 0.3 、 0.4 、 0.5$ 的柱 模拟结果进行对比分析。得出到达屈服荷载之前, 构件的 刚度退化率和耗能能力基本相同。到达屈服荷载后, 随着 轴压比增大, 刚度退化率逐渐增大, 耗能能力逐渐减小, 累积耗能分别减少 $40.84 \%$ 、59.16\%和 $67.18 \%$ 。

张静等 $[8]$ 通过 5 根轴压比 $0.3 \sim 0.65$ 的再生混凝土框架 柱的抗震性能试验, 得出在剪跨比、配箱率等条件一致的 情况下, 随着轴压比增加, 试件的滞回曲线更加扁平, 极
限承载力、延性和滞回耗能不断减小。林颖等[9]以实际 $10.5 \mathrm{~m}$ 高桥墩为例, 通过Opensees建立有限元模型, 得出 轴压比在 $0.1 \sim 0.3$ 范围内, 随轴压比增大, 桥墩承载力不 断增大, 累计耗能最大降低 $41.2 \%$, 位移和延性显著减小。

$\mathrm{Ou}$ 等[10]根据FEMA 461[11]推荐的拟静力试验加载 制度, 研究了考虑长持时和短持时地震动作用下的荷载加 载制度, 并对某桥墩开展了试验分析, 结果表明桥墩纵筋 的屈曲现象应该在分析中有所考虑。杨红等[12-13]考虑屈 曲效应对不同轴压比的RC柱抗震性能的影响, 通过改进 低周疲劳损伤模型, 更加精确地预测了柱纵筋的屈曲, 得 出屈曲的存在以及大应变幅疲劳损伤会加剧钢筋断裂破 坏。

轴压比大小会直接影响混凝土柱的抗震性能, 定量研 究轴压比对柱的滞回性能和延性能力等在结构设计中具 有重要意义。为了全面考虑轴压比对 $\mathrm{RC}$ 框架柱抗震性能 的影响, 本文以某实际 $\mathrm{RC}$ 框架柱为例, 基于拟静力数值 模拟方法, 采用考虑屈曲和疲劳损伤的模型。通过比较 RC 框架柱的滞回曲线、骨架曲线、刚度退化、耗能性能以及 延性等的不同, 重点分析了轴压比对 $\mathrm{RC}$ 框架柱抗震性能 影响。

\section{2. $\mathrm{RC}$ 框架柱基本设计参数}

本文分析的 $\mathrm{RC}$ 框架柱选自某实际3层框架结构, 该结 构为 7 度抗震设防, 抗震等级为三级, 柱截面尺寸为 $450 \mathrm{~mm} \times 450 \mathrm{~mm}$ 。考虑到柱的反弯点处弯矩为零, 因此本 文拟静力数值模拟中进行了简化, 只取半柱进行了建模分 析, 即柱的计算高度为 $1.6 \mathrm{~m}$ 。该 $\mathrm{RC}$ 框架柱纵筋及筢筋均 采用HRB 400 三级钢筋, 靠近基础的柱底部箍筋加密区为 $500 \mathrm{~mm}$, 底梁箱筋间距 $150 \mathrm{~mm}$, 柱纵筋配筋率为 $1.24 \%$, 柱面积配箱率 $0.224 \%$, 体积配箱率 $0.6 \%$ 。混凝土强度等级 为 $\mathrm{C} 30$, 混凝土保护层厚度为 $25 \mathrm{~mm}$ 。框架柱的尺寸及配筋 情况如图1所示。

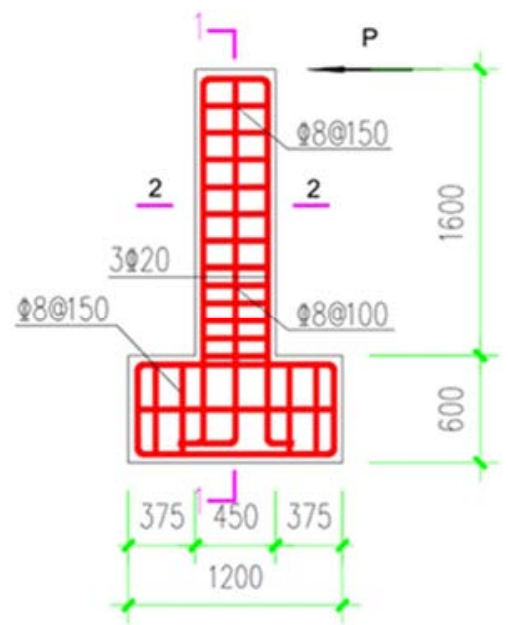

图1 框架柱配筋示意图（单位： $\mathrm{mm}$ ）。 
本文建模中钢筋本构控制参数取张耀庭等[14]单调拉 伸及低周疲劳性能试验中测得的HRB400钢筋数据, 初始 弹性模量 $E_{s}=2 \times 10^{5} \mathrm{Mpa}$, 初始硬化弹性模量
$E_{s h}=6600 M p a$ 。钢筋及混凝土的力学性能指标分别如表 1和表2所示。

表1 钢筋力学性能指标。

\begin{tabular}{lllll}
\hline 钢筋型号 & $\boldsymbol{d}(\mathbf{m m})$ & $\boldsymbol{f}_{\boldsymbol{y}}(\mathbf{M P a})$ & $\boldsymbol{f}_{\boldsymbol{s u}}(\mathbf{M P a})$ & $\boldsymbol{\varepsilon}_{\boldsymbol{s h}}$ \\
\hline 纵筋HRB400 & 20 & 452 & 639 & 0.0164 \\
箍筋HRB400 & 8 & 452 & 639 & 0.134 \\
\hline
\end{tabular}

表1中, $f_{y}$ 为屈服强度, $f_{s u}$ 为极限强度, $\varepsilon_{s h}$ 为初始硬化应变, $\varepsilon_{s u}$ 为极限应变。

表2 混凝土力学性能指标。

\begin{tabular}{lllll}
\hline 混凝土 & $\boldsymbol{f}_{c u, k}(\mathbf{M P a})$ & $\boldsymbol{f}_{c, m}(\mathbf{M P a})$ & $\boldsymbol{f}_{\boldsymbol{c}}(\mathbf{M P a})$ & $\boldsymbol{f}_{\boldsymbol{t}}(\mathbf{M P a})$ \\
\hline $\mathrm{C} 30$ & 30 & 27 & 14.3 & 4 \\
\hline
\end{tabular}

表2中, $f_{c u, k}$ 为混凝土立方体抗压强度, $f_{c, m}$ 为混凝土 轴心抗压强度, $f_{c}$ 为混凝土棱柱体抗压强度设计值, $f_{t}$ 为 混凝土抗拉强度。

\section{RC框架柱建模及本构关系选取}

针对上述混凝土柱, 采用OpenSees有限元分析软件进 行了建模, 开展了拟静力数值模拟分析。建模分析时, 在 对钢筋和混凝土材料本构进行对比分析后, 本文钢筋本构 关系采用Reinforcing steel本构模型。Reinforcing steel本构 是较为精确的钢筋力学特性模拟模型, 钢筋本构曲线分四 段描述，分别为线弹性阶段、屈服阶段、硬化阶段以及最 后的软化阶段。该模型单调应力-应变本构如图2所示。

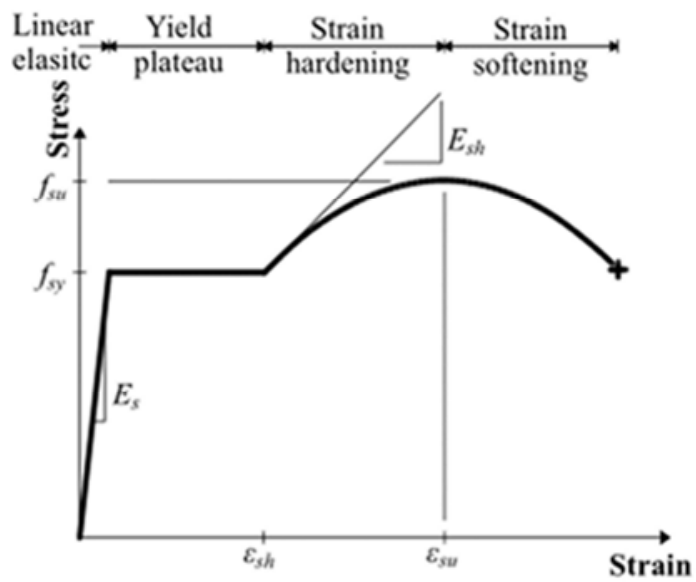

图2 Reinforcing steel单调应力-应变本构。

Reinforcing steel中使用的疲劳-寿命方程[15]如公式 (1) 所示:

$$
\varepsilon_{p}=C_{f}\left(2 N_{f}\right)^{-\alpha}
$$

其中, $\varepsilon_{p}$ 为应变幅值, $C_{f}$ 为由疲劳测试决定的材料常 数, $2 N_{f}$ 为失效前的半循环次数, $\alpha$ 为疲劳指数, $C_{f}$ 越小, 破坏前的循环次数越少。

Reinforcing steel中使用的强度退化方程如公式（2） 所示:

$$
\varepsilon^{P}=C_{d}\left(f_{p}\right)^{-\beta}
$$

其中, $f_{p}$ 为应变为 $\varepsilon^{P}$ 时测得每一循环的强度降低系数, $\beta$ 和 $C_{d}$ 为材料系数, $C_{d}$ 越小, 强度退化越大。

文中取张耀庭等[14]通过试验得的到疲劳损伤参数 $C_{f}=0.140 、 C_{d}=0.379 、 \alpha=0.379$ 。根据Opensees官网资料 [16], 本文取屈曲模型参数 $\beta=1.0 、 \gamma=0.5 、 r=0.4$, 其中 $\beta$ 为屈曲 应力应变曲线的放大系数, 不考虑屈曲时放大系数无影响。 $\mathrm{r}(0<\mathrm{r}<1)$ 用于定义钢筋屈曲的程度, $\mathrm{r}=1$ 表示不考虑屈曲的 影响, $r=0$ 其结果接近传统 $\mathrm{GA}$ 模型[17]的屈曲骨架曲线。

未约束混凝土采用Concrete 02模型, 保护层混凝土受 压峰值强度 $f_{c 0}$ 为 $27 \mathrm{Mpa}$, 峰值应变 $\varepsilon_{c 0}$ 为 0.002 , 极限强度 $f_{u}$ 为 $8 \mathrm{Mpa}$, 考虑混凝土的剥落 [18], 也可取 0 , 极限应变 $\varepsilon_{c u}$ 为 0.01。Concrete 02模型的单调应力-应变本构模型如图3所 示。

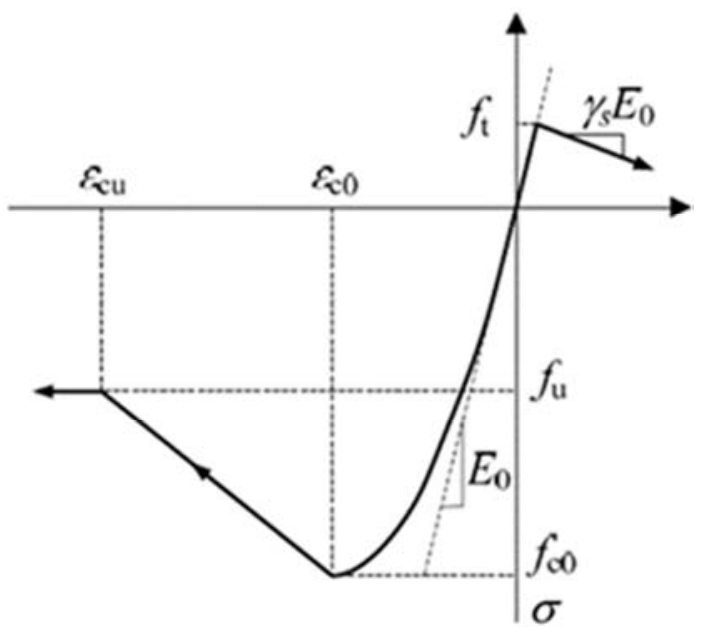

图3 Concrete 02 单调应力-应变本构。

箍筋约束混凝土采用Mander[19]模型, 该模型由 Mander等完成的 31 根不同截面形式的钢筋混凝土柱轴心 受压试验得到, 试验中考虑了约束混凝土面积、筢筋间距、 䈐筋屈服强度以及体积配箍率等的影响。根据Mander模型 计算得出, 文中䈐筋约束混凝土受压峰值强度 $f_{c}$ 为 $34 \mathrm{MPa}$, 峰值应变 $\varepsilon_{c 0}$ 为 0.004 , 极限强度 $f_{c u}$ 为 $5.2 \mathrm{Mpa}$, 极限应变 $\varepsilon_{c u}$ 为 0.04 。 
本文拟静力模拟中, 采用位移控制加载方式, 每个位 移幅度加载一圈, 位移增量为 $5 \mathrm{~mm}$, 加载制度如图 4 所示。

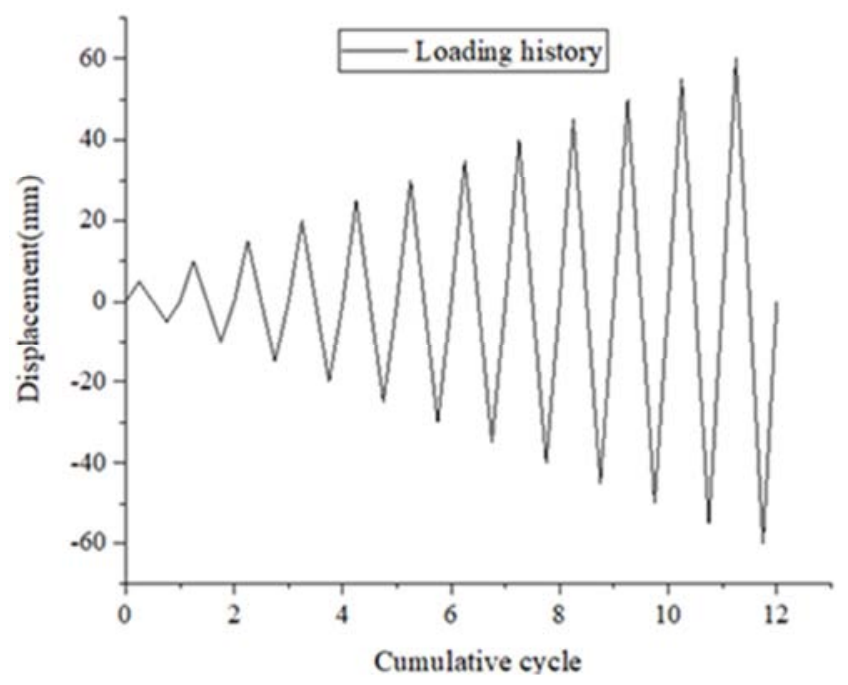

图4 位移控制阶段加载制度。

为了研究柱轴压比的影响, 本文设置了 4 种加载工况, 将柱的轴压比分别设置为 $0.30 、 0.40 、 0.60$ 及 0.80 , 分别模 拟柱轴压比从小到大的情况, 具体的施加坚向轴力及轴压 比如表3所示。

表3 轴压比的确定。

\begin{tabular}{lll}
\hline 工况编号 & 轴力 $(\mathbf{k N})$ & 轴压比 \\
\hline $\mathrm{Z} 1$ & 2316.6 & 0.80 \\
$\mathrm{Z} 2$ & 1734.5 & 0.60 \\
$\mathrm{Z3}$ & 1158.3 & 0.40 \\
$\mathrm{Z4}$ & 868.7 & 0.30 \\
\hline
\end{tabular}

\section{4. 模拟结果分析}

\section{1. 轴压比对滞回曲线影响}

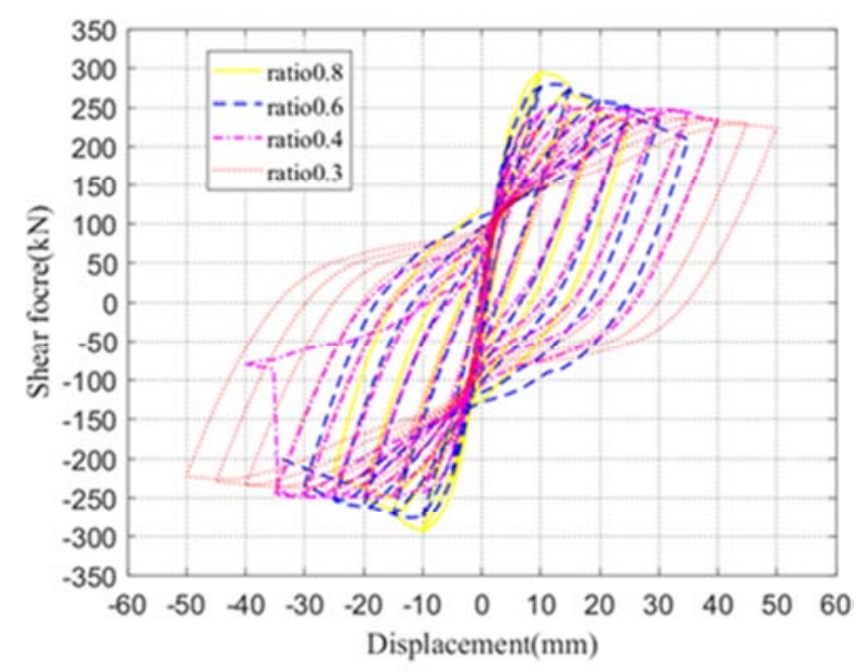

图5 不同轴压比下柱滞回曲线对比。

根据拟静力模拟结果, 不同轴压比下柱的滞回曲线对 比如图5所示。由图5可知, 柱的轴压比越大, 其极限承载
力越大, 滞回曲线变得越来越扁平, 逐步出现“捏缩”现象, 最终成弓形; 柱子的抗侧刚度越大, 骨架曲线的下降段越 来越陡, 位移延性则明显降低。因此, 在 RC框架结构设 计中, 十分有必要限制轴压比的取值范围, 不要将柱的轴 压比取值过大, 以免在地震中发生脆性破坏。

\section{2. 轴压比对骨架曲线影响}

一般情况下，柱的骨架曲线为滞回曲线的包络线，也 可表述为滞回曲线上同向 (拉或压) 各次加载的荷载极值 点依次相连得到的包络曲线称为骨架曲线。通过对图 5 中 模拟结果滞回曲线分析可知, 不同轴压比下, 在两个方向 骨架曲线均呈现出 S型, 本文取上半部分分析, 提取的柱 滞回曲线骨架曲线如图6所示。

由图6可知, 当施加位移荷载较小时, 曲线斜率较大, 说明柱的刚度较大, 随着荷载进一步增大, 骨架曲线的 斜率增加较为平缓, 最后进入下降段。说明柱在往复荷 载作用下经历了弹性、弹塑性和塑性破坏三个受力阶段。 而在弹性阶段, 4 个轴压比下滞回曲线基本重合, 说明在 弹性范围内, 柱的侧向承载力基本不受轴压比的影响。 当轴压比为 0.3 时, 柱的极限承载力为 $236 \mathrm{kN}$, 当轴压比 为 0.8 时, 柱的极限承载力为 $294 \mathrm{kN}$, 柱的最大承载力增 大了 $24.6 \%$ 。因此柱的极限承载力会随着轴压比的增加而 增大, 但轴压比越大, 柱的承载力随位移增加下降速度 越快。

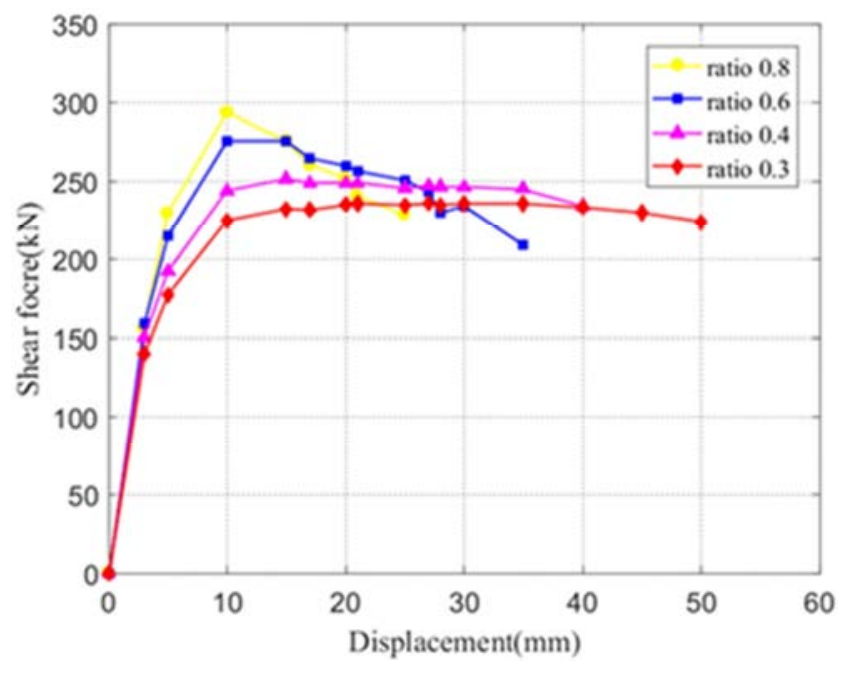

图6 不同轴压比下柱骨架曲线对比。

\section{3. 轴压比对刚度退化曲线影响}

刚度退化是材料塑性变形发展的充分体现, 刚度定义 为各级第一循环的滞回曲线峰值点荷载值与对应位移值 之比。通过对模拟结果分析, 得到的不同轴压比下柱刚度 退化曲线对比如图7所示。

由图可知, 在柱进入弹塑性阶段后, 随着位移增加, 各轴压比下刚度开始退化, 直至最终破坏。轴压比取值越 大, 柱的刚度退化的越来越快, 表明 $\mathrm{RC}$ 柱的塑性变形能 力随着轴压比的增大变差。 


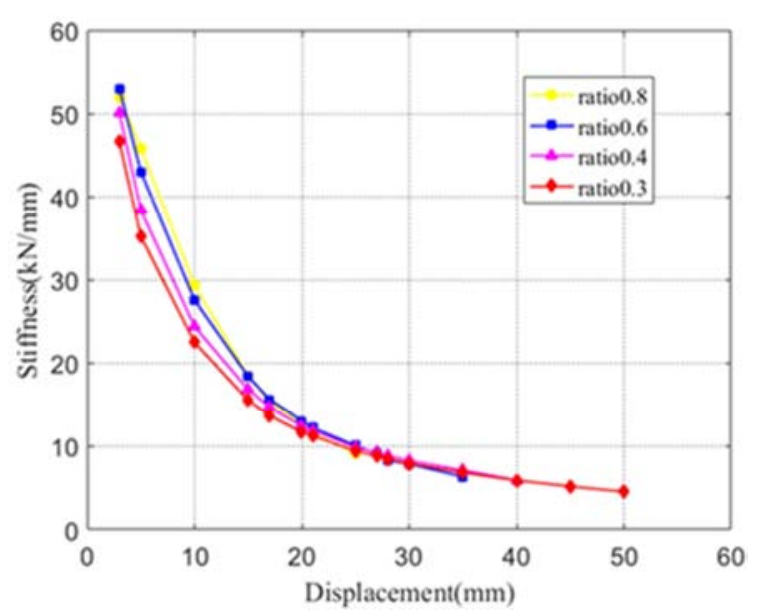

图7 不同轴压比下柱刚度退化曲线对比。

\section{4. 轴压比对耗能能力影响}

根据滞回耗能的定义, 柱在每级加载下的耗能可以等 效为相对应滞回环的面积。可以采用等效粘性阻尼[20]的 表示耗能, 等效粘性阻尼越大, 柱的塑性程度越严重, 即 柱的破坏程度越严重, 其表达式如公式（3）所示:

$$
h_{e}=\frac{E_{d}}{2 \pi K_{\sec } \Delta_{o}^{2}}
$$

其中: $E_{d}$ 是每个加载周期消耗的总能量, $K_{s e c}$ 是割线 刚度, $\Delta_{o}$ 是循环的幅度。对本文框架柱而言, 分析结果如 图8所示。

由图8可知, 随加载位移幅值增大, 等效粘性阻尼比 不断增大。在未超过极限变形时, 同一位移幅值下, 高轴 压比下的柱等效粘性阻尼比更大, 即同一幅值加载下柱的 单圈耗能较大。轴压比从 0.6 增大到 0.8 时, 等效粘滞阻尼 比的变化率更大。

把柱每级荷载下滞回环的面积相加可以得到结构的 总耗能, 不同轴压比下柱的总耗能如图9所示。可以看出, 轴压比从 0.3 增加到 0.8 时, 累积耗能值从 $85.4 \mathrm{kN} \cdot \mathrm{m}$ 降低到 $30.5 \mathrm{kN} \cdot \mathrm{m}$, 降低幅度为 $64.2 \%$, 即随着轴压比增大, 柱的 耗能能力越来越差。

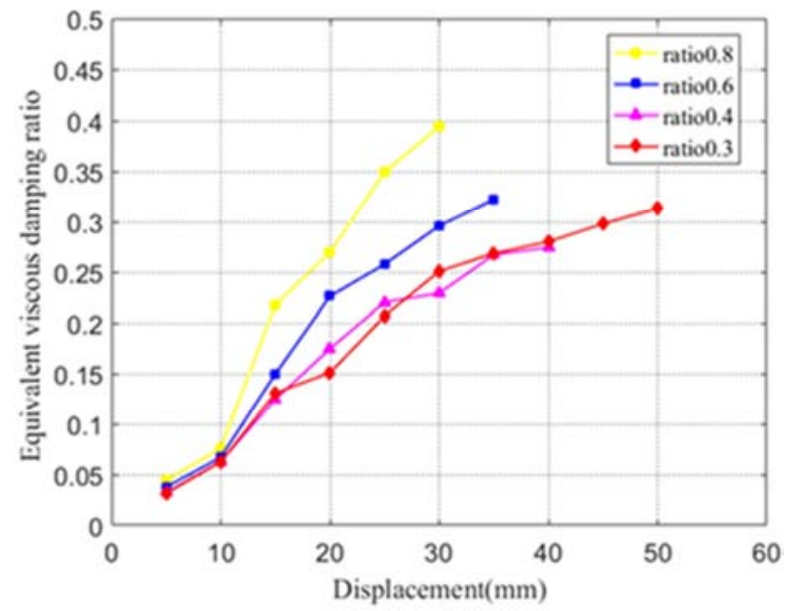

图8 不同轴压比下等效粘性阻尼比对比。

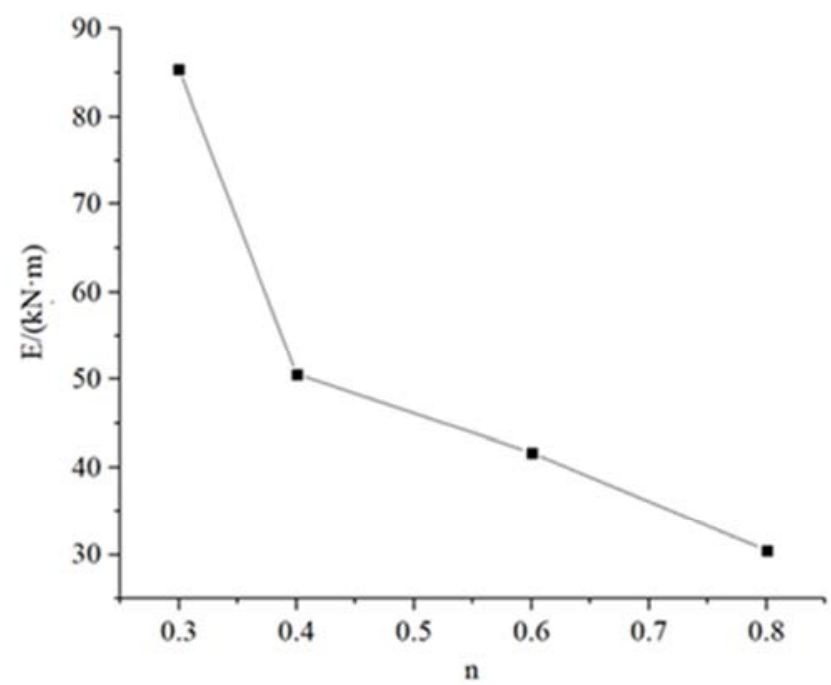

图9 不同轴压比下柱总耗能对比。

\section{5. 轴压比对延性能力影响}

定义柱的延性系数 $u$ 为极限位移 $\Delta_{u}$ 与屈服位移 $\Delta_{y}$ 的比 值, $F_{y}$ 为屈服荷载, $F_{u}$ 为极限荷载。

表4 柱位移延性系数计算结果。

\begin{tabular}{llllll}
\hline 轴压比 & $\boldsymbol{F}_{\boldsymbol{y}}(\mathbf{k N})$ & $\Delta_{\boldsymbol{y}}(\mathbf{m m})$ & $\boldsymbol{F}_{\boldsymbol{u}}(\mathbf{k N})$ & $\Delta_{\boldsymbol{u}}(\mathbf{m m})$ & $\boldsymbol{u}$ \\
\hline 0.3 & 212.4 & 7.3 & 200.6 & 50.0 & 6.8 \\
0.4 & 226.7 & 7.2 & 214.3 & 40.0 & 5.6 \\
0.6 & 250.6 & 6.8 & 236.7 & 28.0 & 4.1 \\
0.8 & 260.2 & 6.6 & 250.0 & 20.0 & 3.0 \\
\hline
\end{tabular}

由表 4 可知, 轴压比从 0.3 增大到 0.8 , 柱的屈服位移不 断降低, 但变化的幅度不大, 极限位移不断降低, 降低的 幅度为最大为 $60 \%$; 屈服荷载和极限荷载不断增大, 增大 的幅度分别为 $22.5 \%$ 和 $24.6 \%$; 柱的位移延性系数不断减小, 轴压比为 0.8 时相较轴压比为 0.3 时, 柱的延性系数降低了 $55.9 \%$, 说明轴压比增大, 柱的延性能力会降低。

\section{5. 结论}

本文采用OpenSees有限元软件，对某RC框架柱进行 了拟静力数值模拟, 分析中采用了考虑纵筋屈曲和疲劳损 伤的模型, 主要分析了轴压比对柱滞回曲线、刚度、耗能 以及延性的影响, 得到如下结论:

（1）柱轴压比在 $0.3 \sim 0.8$ 范围内, 随着轴压比增加, 柱的滞回曲线变得越来越扁平, 峰值承载力不断增大, 最 大可增大 $24.6 \%$, 但柱承载力下降速度也越快, 刚度退化 越来越快。

(2) 同一位移幅值下, 高轴压比柱的等效粘滞阻尼 更大, 但到达破坏状态时, 低轴压比构件的总耗能明显大 于高轴压比构件, 轴压比从 0.3 增大到 0.8 时, 构件累积耗 能值降低了 $64.2 \%$ 。

(3) 轴压比的增大会降低柱的屈服位移和极限位移, 会增大屈服荷载和极限荷载, 轴压比为 0.8 时柱延性系数 比轴压比为 0.3 时的延性系数降低了 $55.9 \%$ 。 


\section{致谢}

本文为国家重点研发计划项目课题 《城市工程强震反 应观测新型传感器及组网观测技术》 (2017YFC1500601) 及其省级资助项目（GX18C005）与国家自然科学基金面 上项目 《基于贝叶斯估计的 $\mathrm{RC}$ 框架结构地震损伤概率识 别方法改进与验证》（51678541）的阶段性成果之一。

\section{参考文献}

[1] 陆新征, 叶列平, 潘鹏, 等. 钢筋混凝土框架结构拟静力倒 塌试验研究及数值模拟竞赛 II : 关键构件试验 [ ] ]. 建筑结构, 2012, 42(11): 23-26.

[2] Yao D L, Ma Y C, Jia J Q. The Influence of Axial Compression Ratio on Seismic Behavior of SRUHSC Frame under Cyclic Loading[J]. KSCE Journal of Civil Engineering,2019,23(2).

[3] 白巨巨, 李升才. 不同轴压比RCS梁柱组合件抗震性能分 析[J].中南大学学报(自然科学版),2020,51(8):2183-2196.

[4] 蔡健, 段伟宁, 汤序霖,等. 轴压比与剪跨比对带约束拉杆 双层钢板-混凝土组合剪力墙抗震性能影响研究 [J].建筑结 构学报,2018,39(2):37-43.

[5] 冯宝锐. 钢筋混凝土柱抗震性能点转角研究[D]. 清华大学, 2014.

[6] Sun L, Yang Z Y, Jin Q, et al. Effect of Axial Compression Ratio on Seismic Behavior of GFRP Reinforced Concrete Columns[J]. International Journal of Structural Stability and Dynamics,2020,20(6).

[7] Liu M, Yin S P, Cong X. Seismic behavior of textile-reinforced concrete-strengthened RC columns under different axial compression ratios[J]. Journal of Engineered Fibers and Fabrics,2019,14.

[8] 张静, 周安, 柳炳康, 等. 不同轴压比再生混凝土框架柱抗 震性能试验研究 [J]. 合肥工业大学学报(自然科学版), 2012, 35(4): 503-507.
[9] 林颖, 李刚. 轴压比对钢筋混凝土桥墩抗震性能的影响分 析[J]. 北方交通, 2020, 322(2): 16-19.

[10] Ou Y C, Song J, Wang P H, et al. Ground Motion Duration Effects on Hysteretic Behavior of Reinforced Concrete Bridge Columns[J]. Journal of Structural Engineering,2013.

[11] FEMA 461, Interim Testing Protocols for Determining the Seismic Performance Characteristics of Structural and Nonstructural Components (FEMA 461) [S], Applied Technology Council for the Federal Emergency Management Agency, Washington, DC, USA, 2007.

[12] 杨红, 张洛, 张和平. 考虑纵筋屈曲及疲劳损伤的钢筋混 凝土柱抗震性能试验研究与非线性分析 [J]. 建筑结构学 报,2013,34(11):130-140.

[13] 杨红, 再小峰, 谢琴. 考虑屈曲效应的混凝土柱纵筋低周 疲劳性能和变形能力研究 [J]. 建筑结构学报, 2021, 42(3): 105-116.

[14] 张耀庭, 赵璧归, 李瑞鸽,等. HRB400钢筋单调拉伸及低周 疲劳性能试验研究 [J]. 工程力学, 2016, 33(4): 121-129.

[15] Brown J, Kunnath S K. Low Cycle Fatigue Behavior of Longitudinal Reinforcement in Reinforced Concrete Bridge Columns[R]. University at Buffalo, State University of New York, Technical Report MCEER-00-0007, 2000.

[16] Mckenna F, Fenves G L, Scott M H. Open System for Earthquake Engineering Simulation. Berkeley, CA:University of California, Available from: http: / /OpenSees berkeley edu, 2000 .

[17] Gomes A, Appleton J. Nonlinear Cyclic Stress-Strain Relatinship of Reinforcing Bars Including Buckling[J]. Engineering Structures, 1997, 19(10): 822-826.

[18] 李雁军, 吕大刚, 王震宇, 等. 钢筋混凝土框架结构拟静力 倒塌实验数值模拟 [J]. 工程力学, 2012, 29(S2): 205-209.

[19] Mander J A B, Priestley M J N. Theoretical Stress-Strain Model for Confined Concrete[J]. Journal of Structural Engineering, 1988, 114(8): 1804-1826.

[20] Ou Y C, Wang P H, Tsai M S, et al. Large-Scale Experimental Study of Precast Segmental Unbonded Posttensioned Concrete Bridge Columns for Seismic Regions[J]. Journal of Structural Engineering, 2010, 136(3): 255-264. 